\title{
Hericium erinaceus mycelium ameliorate anxiety induced by continuous sleep disturbance in vivo
}

Tsung-Ju Li ${ }^{1}$, Tung-Yen Lee ${ }^{2}$, Yun Lo ${ }^{2}$, Li-Ya Lee ${ }^{1}$, I-Chen Li ${ }^{1}$, Chin-Chu Chen ${ }^{1,3,4^{*}}$ and Fang-Chia Chang $2,5,6,7^{*}$

\begin{abstract}
Background: Sleep disruption is a major public health issue and may increase the risk of mortality by ten-folds if an individual is sleeping less than $6 \mathrm{~h}$ per night. Sleep has changed dramatically during to the COVID-19 pandemic because COVID symptoms can lead to psychological distress including anxiety. Hericium erinaceus mycelium has been widely investigated in both the in vivo studies and clinical trials for its neuroprotective functions because the mycelium contains hericenones and erinacines, which synthesize the nerve growth factor and brain-derived neurotrophic factor (BDNF). Recent in vivo reports have shown showed that erinacine A-enriched Hericium erinaceus mycelium can modulate BDNF/TrkB/PI3K/Akt/GSK-3 $\beta$ pathways to induce an antidepressant-like effect. A large body of evidence indicates that erinacine can pass the blood-brain barrier and suggests its neuroprotective function in both peripheral and central nervous systems. Thus, Hericium erinaceus mycelium may be a dual-function supplement for sleep disruption improvement while sustaining anxiolytic effects.
\end{abstract}

Method: To simulate the condition of sleep disruption, the mice were subjected to the tail suspension test (TST) for $15 \mathrm{~min}$ every day during the same period for nine consecutive days. Two different doses $(75 \mathrm{and} 150 \mathrm{mg} / \mathrm{kg}$ ) of Hericium erinaceus mycelium were administered orally $20 \mathrm{~min}$ prior to the TSTs before entering the light period of 12:12 h L:D cycle. All sleep-wake recording was recorded for $24 \mathrm{~h}$ using electroencephalogram and electromyogram. The elevated-plus-maze and open-field tests were conducted to record the behavior activities.

Results: Consecutive TSTs prior to the light period could cause significant sleep disturbance and anxiety behavior in the elevated-plus-maze experiments. Results showed that administration with Hericium erinaceus mycelium at $150 \mathrm{mg} / \mathrm{kg}$ ameliorated the rodent anxiety $(p<0.05)$ and reversed the TST-induced NREM sleep disturbance in the dark period.

Conclusion: This is the first in vivo study suggesting that Hericium erinaceus mycelium has a dual potential role for anxiety relief through improving sleep disruptions.

Keywords: Hericium erinaceus mycelium, COVID-19, Sleep, Anxiety, NREM

*Correspondence: gkbioeng@grapeking.com.tw; fchang@ntu.edu.tw

2 Department of Veterinary Medicine, National Taiwan University, Taipei, Taiwan

${ }^{4}$ Institute of Food Science and Technology, National Taiwan University,

Taipei, Taiwan

Full list of author information is available at the end of the article

\section{Background}

Sleep deprivation is an important public issue in many countries. Statistics have shown that approximately 83.6 million adults in the United States sleep less than $7 \mathrm{~h}$ a day [1]. Sleep deprivation often causes a lack of energy restoration and metabolite clearance in the brain, which is crucial in sustaining brain function and behaviors [2]. 
Poor sleep quality as defined by the National Sleep Foundation [3], can often occur from working pressure, stress, anxiety, and financial burden; it eventually affects the formation of long-term memory [4]. Sleep deprivation is believed to be closely related to anxiety levels [5]. Nevertheless, the recent pandemic issue of COVID-19 causing stress and anxiety at the same time have greatly altered the sleep quality [6].

Sleep is divided into two distinct states: rapid eye movement (REM) sleep and non-rapid eye movement (NREM) sleep [5, 7]. A study showed that changes in NREM sleep often correlate with anxiety levels in mice [8]. On the other hand, REM sleep latency is a feature of the early symptoms in depression [9]. Nerve growth factor (NGF) is a key and important modulator in suppressing wakefulness and contributes to the generation of REM sleep [10]. Nevertheless, once anxiety symptoms occur from sleep deprivation, this kind of vicious sleep disruption will hamper the therapeutic efficacy in anxiety patients. Therefore, proper and safe supplementary nutrients are needed for anxiety relief while improving sleep patterns in anxious patients [11].

Hericium erinaceus (H. erinaceus) has long been used as a medicinal-culinary mushroom [12], which showed various health benefits including anti-aging [13], antioxidative [14], anticancer [15], and neuroprotection [16]. Studies found that the H. erinaceus cultured extract contains about more than 15 compounds of the active cyathin diterpenoid [17] with potential neuroprotective effects in the treatments of Alzheimer's and Parkinson's diseases [18]. Incubation with $H$. erinaceus erinacine compounds increases NGF gene expression in the human astrocytoma cell line 1321 N1 [19]. Erinacine A-enriched $H$. erinaceus mycelium can induce an antidepressant-like effect by modulating the BDNF/TrkB/PI3K/Akt/GSK-3 $\beta$ pathway in vivo [20]. An in vivo biodistribution study found that erinacine from the $H$. erinaceus mycelium can be found in the brain tissue indicating its capability to pass the blood-brain barrier and exert a neuroprotective function [21]. A double-blind placebo-controlled study further showed that consumption of three $H$. erinaceus mycelium capsules per day (containing $5 \mathrm{mg} / \mathrm{g}$ of erinacine A) can prevent early Alzheimer's disease versus a placebo group [22]. All these studies suggest that $H$. erinaceus mycelium is safe and effective at improving life quality for patients with neurodegeneration.

Despite the accumulated evidence that $H$. erinaceus has in terms of anti-depression and memory improvement [23], there is no report to date that has investigated the potential of $H$. erinaceus mycelium for sleep deprivation with early anxiety symptoms. The aim of this study is to investigate the alterations of behavior activities and monoamines in a sleep disrupted mouse model treated with nutrient $H$. erinaceus mycelium. We then evaluated its potential as an anxiety relief supplement that can improve sleep.

\section{Methods \\ Preparation of the $\boldsymbol{H}$. erinaceus mycelium}

The $H$. erinaceus mycelium was prepared according to previous studies [24]. In brief, the $H$. erinaceus strain was purchased from the Bioresources Collection and Research Center in the Food Industry Research and Development Institute (BCRC 35669; Hsinchu, Taiwan). Initial culture was grown in a 2 -L flask using a shaker at $\sim 120 \mathrm{rev} / \mathrm{min}$ at $25^{\circ} \mathrm{C}$ for 5 days containing growth medium at $\mathrm{pH} 4.5\left(0.05 \% \mathrm{MgSO}_{4}, 0.25 \%\right.$ peptone, $0.5 \%$ soybean powder, $4.5 \%$ glucose, and $0.25 \%$ yeast extract). The seeding medium is then transferred to $500-\mathrm{L}$ fermenters for 5 days and scaled up to 20-ton fermenters for another 12 consecutive days. This large-scale process is then harvested and lyophilized to remove excessive water. The final product is ground into powder form and stored in desiccators at room temperature for in vivo experiments. To evaluate the main chemical composition of Hericium erinaceus Mycelium, high-performance liquid chromatography (HPLC) was used to evaluate the active peaks' contents. Major composition such as erinacine $\mathrm{A}$ and erinacine $\mathrm{C}$ were analyzed according to previous studies with minor modifications $[24,25]$.

\section{Feeding condition}

All animal experiments were carried out in compliance with the ARRIVE guidelines and were approved by the National Taiwan University Institutional Animal Care and Use Committee (approval number NTU107-EL-00182). The C57BL/6 mice used in this study were obtained from BioLASCO (TaiwanCo., Ltd.). Oral gavage was employed for the drug delivery. Two different doses of $H$. erinaceus mycelium (75 and $150 \mathrm{mg} / \mathrm{kg}$ ) were administered. All administrations of substances were administered $20 \mathrm{~min}$ prior to the tail suspension test (TST) before entering the light period of 12:12 h L:D cycle.

\section{Tail suspension test (TST)}

The TST was executed from the beginning of the light period and lasted for $15 \mathrm{~min}$ every day and the procedure was performed for nine consecutive days during the same time point. Noise and light were avoided while the tail suspension test experiment was conducted. The environment background was set to the white color during the behavioral task to enhance the contrast of the mice. During the TSTs, the struggle movement was determined 
offline by EthoVision XT software (Noldus Information Technology, Inc., USA). The TST is one of the most widely used models to assess depression in rodents. Previous studies have shown that the TSTs is an acute stressor that causes sleep disruptions in mice [26].

\section{Elevated plus maze (EPM) behavior test}

The EPM was conducted with a similar method as described previously [20]. In brief, the apparatus was placed at a height $50 \mathrm{~cm}$ above the floor with two open arms $(50 \mathrm{~cm} \times 9 \mathrm{~cm})$ and two enclosed arms $(50 \mathrm{~cm} \times 9 \mathrm{~cm} \times 5 \mathrm{~cm})$. The common central area is $9 \times 9 \mathrm{~cm}$. Time spent in both open and closed arms was recorded by the video camera. All paths were wiped with $70 \%$ ethanol between each individual test to avoid disturbance due to the scent of the previously tested animal.

\section{Open field test (OF)}

The open field test (OF) was conducted in an acrylic box $(60 \mathrm{~cm} \times 60 \mathrm{~cm} \times 20 \mathrm{~cm})$ with a floor divided equally with 96 squares. A digital video camera was fixed vertically above the cage to record the mouse activities. For every mouse analysis, the cage was cleaned with water to remove scent of the previously tested animal to prevent any bias.

\section{Sleep recording and analysis}

Two wire electroencephalogram (EEG) electrodes were surgically implanted on the right frontal lobe and the left occipital lobe. The occipital electrode served as the reference. Two electromyogram (EMG) electrodes were inserted into the neck muscle. The sleep-wake activity was recorded for $24 \mathrm{~h}$ after the manipulations. Based on the previous study [27], the animal's vigilance states were classified to NREM sleep, REM sleep, or wakefulness. Briefly, NREM sleep was characterized by large-amplitude EEG slow waves, high power density values in the delta frequency band $(0.5-4.0 \mathrm{~Hz})$, and a relaxed muscle tone from EMGs. During REM sleep, the amplitude of the EEG was reduced, and the predominant EEG power density occurs within the theta frequency $(6.0-9.0 \mathrm{~Hz})$. The EMGs exhibit muscle atonia with low EMG amplitudes.

The animals are generally active during wakefulness. There are protracted body movements with robust EMG amplitudes. The amplitude of EEGs is like that observed during REM sleep, but power density values in the delta frequency band are generally greater than those in theta frequency band. The percentage of NREM sleep and REM sleep, slow wave activity during the NREM sleep, and sleep architectures were determined. Animals were housed in individual recording cages in an isolated animal room with the temperature maintained at $23 \pm 1{ }^{\circ} \mathrm{C}$ and a light:dark (L:D) cycle of 12:12-h $(20 \mathrm{~W} \times 6$ tubes illumination). Food and water were available ad libitum.

\section{Plasma dopamine analysis}

After the behavioral tests, all mice were sacrificed by perfusion with carbon dioxide $\left(\mathrm{CO}_{2}\right)$ and decapitation. The blood was quickly obtained from the mouse's heart and stored at $-80^{\circ} \mathrm{C}$ until the dopamine levels were measured. The enzyme-linked immunosorbent assay (ELISA) kit for dopamine was obtained from Wuhan Fine Biotech Co., and the detailed procedure followed the manufacturer's instructions. The absorbance was determined by ELISA microplate (Multiskan EX, Thermo Electron Corp., Waltham, MA) with an O.D. absorbance at $450 \mathrm{~nm}$. The sensitivity is $<0.938 \mathrm{ng} / \mathrm{ml}$, and the assay range is between 1.56 and $100 \mathrm{ng} / \mathrm{ml}$. The intra-assay $\mathrm{CV}$ is $<8 \%$ and the inter-assay $\mathrm{CV}$ is $<10 \%$ (manufacturer's specifications).

\section{Western blot analysis}

The mouse brain tissue was homogenized with RIPA buffer containing phosphatase and protease inhibitors. The total protein content was quantified using Pierce ${ }^{\mathrm{TM}}$ BCA Protein Assay Kit $(23,227$, Thermo, USA). Protein lysates were separated with $10 \%$ SDS-PAGE gel by electrophoresis and transferred to polyvinylidene difluoride (PVDF) membranes (ISEQ00010, Millipore, USA) using electroblotting transfer tank (Bio-Rad). The PVDF membrane was blocked with $5 \%$ non-fat milk powder in tris-buffered saline with Tween 20 (TBST). Primary antibodies, including BDNF (ab108319; Abcam) and GAPDH (SC-32233; Santa Cruz Biotechnology), were incubated with the membranes diluted at $4{ }^{\circ} \mathrm{C}$ overnight. Secondary antibody $(65-6120,62-6520$; Invitrogen) was applied and the bound-protein bands were visualized using enhanced chemiluminescence (K-12045-D50, Advansta, USA) and quantified using detection system (BIO-RAD ChemiDoc XRS+, USA). The target protein relative intensity was normalized against GADPH.

\section{Statistical analysis}

The percentages of time spent in NREM sleep, REM sleep, the immobility during the TSTs, the open arm during EPM, and the inner area during the OF were represented as the mean \pm standard error of mean (SEM) with indicated sample sizes. This study used 
two-way analysis of variance (ANOVA) to measure the difference between each group with a post-hoc comparison. A level of $p<0.05$ was considered to indicate a statistically significant difference.

\section{Results}

The effects of the consecutive TSTs on sleep-wake activity The consecutive 9-day TSTs resulted in an increase in animal immobilization by $40 \%$ (Fig. 1) demonstrating that continuous stress can lead to a more emotional helplessness behavior in depression.

We manipulated the TSTs prior to the light period to further evaluate whether the consecutive TSTs affects sleep-wake activity. The results showed that the TSTs conducted before the light period significantly decreased NREM sleep in the subsequent dark period (hours 13-24) (Fig. 2A), and REM sleep was significantly increased in the late stage of the dark period (hours 20-22) (Fig. 2B).

The analytical results indicate that consecutive TSTs at the beginning of the light period could induce sleep disturbance. The TSTs during the light period could cause a decline in NREM sleep and a rise in the REM sleep in the subsequent dark period.

The effects of $H$. erinaceus mycelium on sleep-wake activity The $H$. erinaceus mycelium was given prior to the light period of a 12:12 h L:D cycle in the TSTs-treated mice. The results showed that both $75 \mathrm{mg} / \mathrm{kg}$ and $150 \mathrm{mg} / \mathrm{kg}$ of $H$. erinaceus mycelium could increase the percentage of time spent in NREM sleep during the dark period.

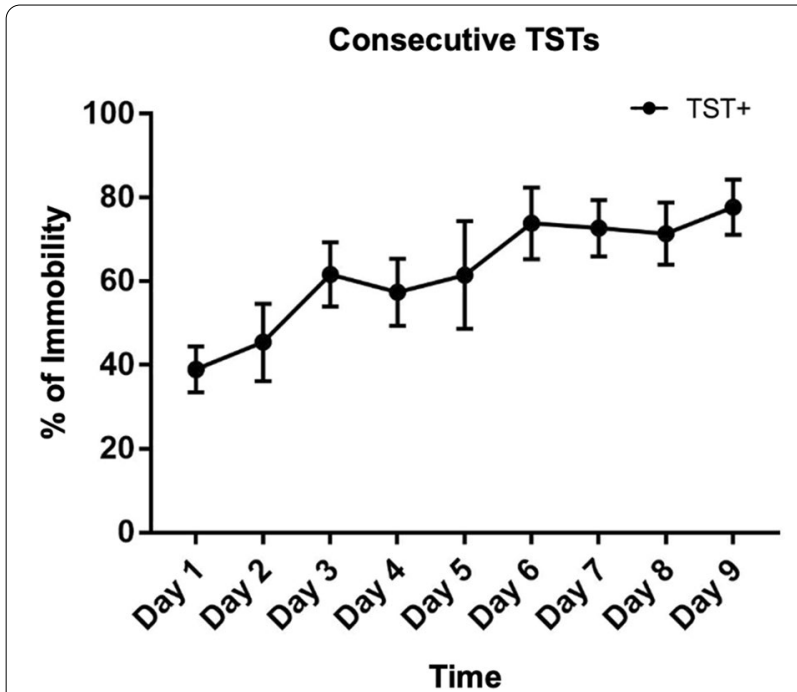

Fig. 1 The mice cumulative immobility analysis after the consecutive 9-day TSTs $(n=6)$
The effect of $H$. erinaceus mycelium on NREM increases showed up at the late stage of the dark period (hours $19-24)$ in the low dose $(75 \mathrm{mg} / \mathrm{kg}$ ) group (Fig. 3A). The rise of NREM sleep appeared nearly during the $12 \mathrm{~h}$ dark period (hours $15-24)$ in the high-dose $(150 \mathrm{mg} / \mathrm{kg}$ ) group (Fig. 3B). Nevertheless, administration of $75 \mathrm{mg} / \mathrm{kg} H$. erinaceus mycelium exhibited no effect on TSTs-induced increase of REM sleep; meanwhile there was a REM sleep decline at hour 20 in the group treated with $150 \mathrm{mg} / \mathrm{kg}$ of $H$. erinaceus mycelium versus the TSTs group (Figs. 3C and D).

These results suggest that the high dose $(150 \mathrm{mg} /$ $\mathrm{kg}$ ) of $H$. erinaceus mycelium could block the TSTsinduced decreases of NREM sleep; it also increased REM sleep in the dark period. However, the low dose (75 mg/kg) of $H$. erinaceus mycelium could only block the TSTs-induced decreases of NREM sleep in the dark period.

\section{The effects of $H$. erinaceus mycelium on EPM activity} In the EPM experiments, $H$. erinaceus mycelium was administered prior to the light period of the 12:12 h L:D cycle. The results showed that $150 \mathrm{mg} / \mathrm{kg}$ of $H$. erinaceus mycelium could increase the proportion of time spent in the open arms compared with that of the TST+ group (Fig. 4). However, lower doses of $H$. erinaceus mycelium $(75 \mathrm{mg} / \mathrm{kg})$ showed no anxiolytic effects.

\section{The effects of $H$. erinaceus mycelium in OF test}

In the OF test experiments, $H$. erinaceus mycelium was administered prior to the light period of the $12: 12 \mathrm{~h} \mathrm{~L}: \mathrm{D}$ cycle. The results showed that $150 \mathrm{mg} / \mathrm{kg}$ of $H$. erinaceus mycelium could increase the proportion of time spent in the open arms compared with that of the TST+ group (Fig. 5). However, lower doses of $\mathrm{H}$. erinaceus mycelium $(75 \mathrm{mg} / \mathrm{kg})$ also showed no anxiolytic effects.

\section{The effects of $H$. erinaceus mycelium in plasma dopamine activity and brain tissue BDNF}

In the plasma neurotransmitter analysis, $H$. erinaceus mycelium was administered prior to the light period of the 12:12 h L:D cycle. When TSTs were conducted, the plasma dopamine levels decreased significantly by $26.5 \%$. Low dose of $H$. erinaceus mycelium has no effect in the recovery of the dopamine levels. However, the high dose of $H$. erinaceus mycelium significantly recovered the plasma dopamine concentrations back to normal levels $(435.882 \pm 32.098 \mathrm{ng} / \mathrm{mL}$, Fig. 6). The 


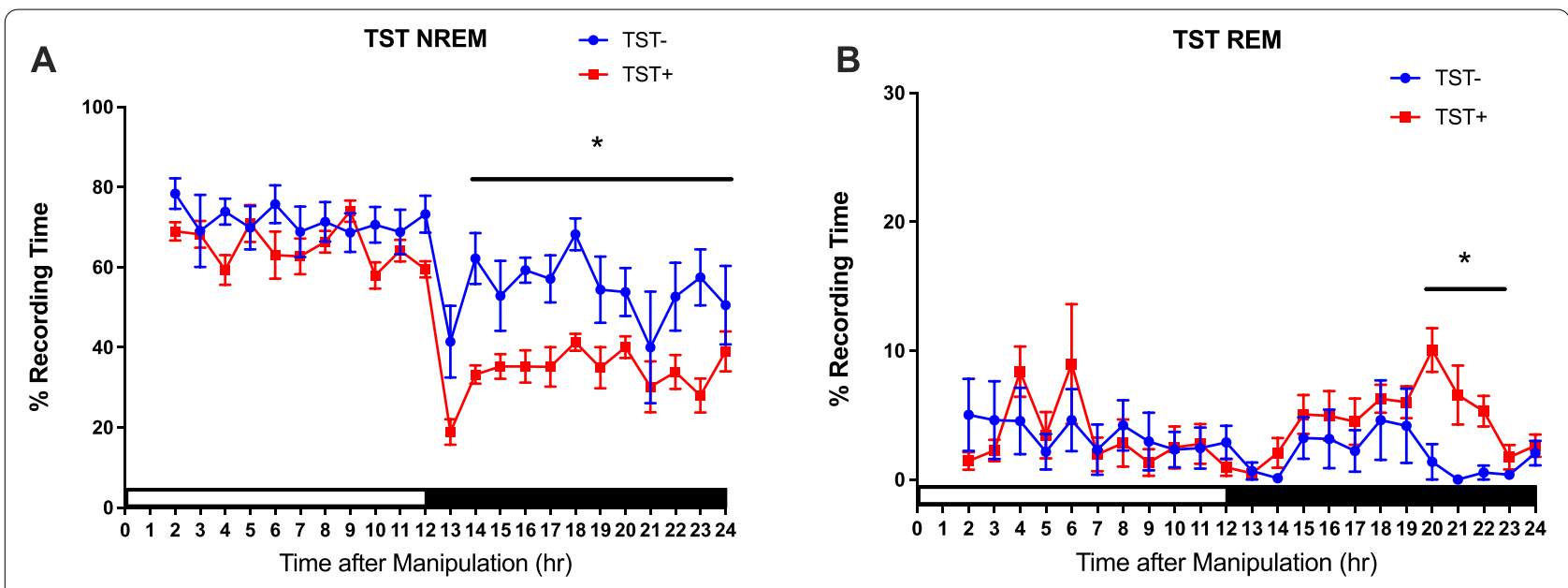

Fig. 2 A The blue circles represent NREM sleep acquired from the control group without the TSTs $(n=6)$, and the red squares represent the data obtained from the group with the TSTs $(n=6)$. The $x$-axis depicts the time after the TSTs, and the $y$-axis represents the percentages of NREM sleep. B The blue circles represent REM sleep acquired from the control group without the TSTs $(n=6)$, and the red squares represent the data from the group with the TSTs $(n=6)$. The $x$-axis depicts the time after the TSTs, and the $y$-axis represents the percentages of REM sleep. The white and black bars demonstrate the $12 \mathrm{~h}$ light period and $12 \mathrm{~h}$ dark period, respectively. All data with a * sign means $p<0.05$

mouse brain BDNF expression was also evaluated by western blot analysis. TSTs group showed a significant reduction of BDNF expression after continuous stress. At high dose of $H$. erinaceus mycelium treatment, an increase in BDNF expression can be observed when compared with the control group (Supplementary Fig. S1).

\section{HPLC analysis of the components from $\mathrm{H}$. erinaceus mycelium}

The identification of the erinacine component was extracted using $85 \%$ ethanol from the $H$. erinaceus mycelium (Fig. 7). The erinacine $\mathrm{A}$ and erinacine $\mathrm{C}$ was confirmed by comparing the characteristics of the spectrum of the sample peak with those of the standard peak (blue line, erinacine A; purple line, erinacine $C$ ) with a yield of $7.20 \mathrm{mg} / \mathrm{g}$ and $3.35 \mathrm{mg} / \mathrm{g}$, respectively.

\section{Discussion}

Insufficient sleep has become a public health issue according to the US Centers for Disease Control and Prevention (CDC) [28]. Individuals who sleep less than $6 \mathrm{~h}$ have a ten-fold increased mortality [29]. Moreover, these issues might have changed dramatically due to the recent COVID-19 pandemic. Researchers have found that COVID-19 is closely associated with individual psychological distress [30]. Of the 1250 self-isolated healthcare professionals during the outbreak, $44.6 \%$ of them reported reducing sleep quality because of high anxiety levels [31]. Thus, new tools are needed to reduce anxiety and promote sleep especially in those affected by COVID-19.

Several studies have shown that $H$. erinaceus mycelium can stimulate the synthesis of NGF for neuroprotection and prevents neurodegenerative diseases [32]. Depression is a common neuropsychiatric conditions and abnormal behaviors can be improved by the $H$. erinaceus mycelium in animals [33]. Sleep disturbance is a complicated mechanism, and the TST is a useful method to evaluate sleep deprivation [34]. By using the TST-induced depression model, we showed that the consecutive 9-day TSTs stress performed at the beginning of the light period could generate a significant sleep disturbance while concurrently causing helplessness behavior. Based on the idea that sufficient NREM sleep is essential for brain development and functions [35], we evaluated whether $H$. erinaceus mycelium treatment can ameliorate sleep disturbance in the TSTs-induced depressive animals. Versus TST-induced models without any medication treatment, our results showed that a high dose of $H$. erinaceus mycelium blocked TSTs-induced increases of NREM sleep.

The REM analysis literature has shown that stressinduced sleep deprivation can cause a REM sleep rebound in the dark period [36]. We also observed this REM sleep rebound after consecutive TSTs. We also found that this TSTs-induced REM sleep rebound was reversed back to normal when $H$. erinaceus mycelium was treated. Administration of $H$. erinaceus mycelium at $150 \mathrm{mg} / \mathrm{kg}$ alleviates the stress-induced sleep 


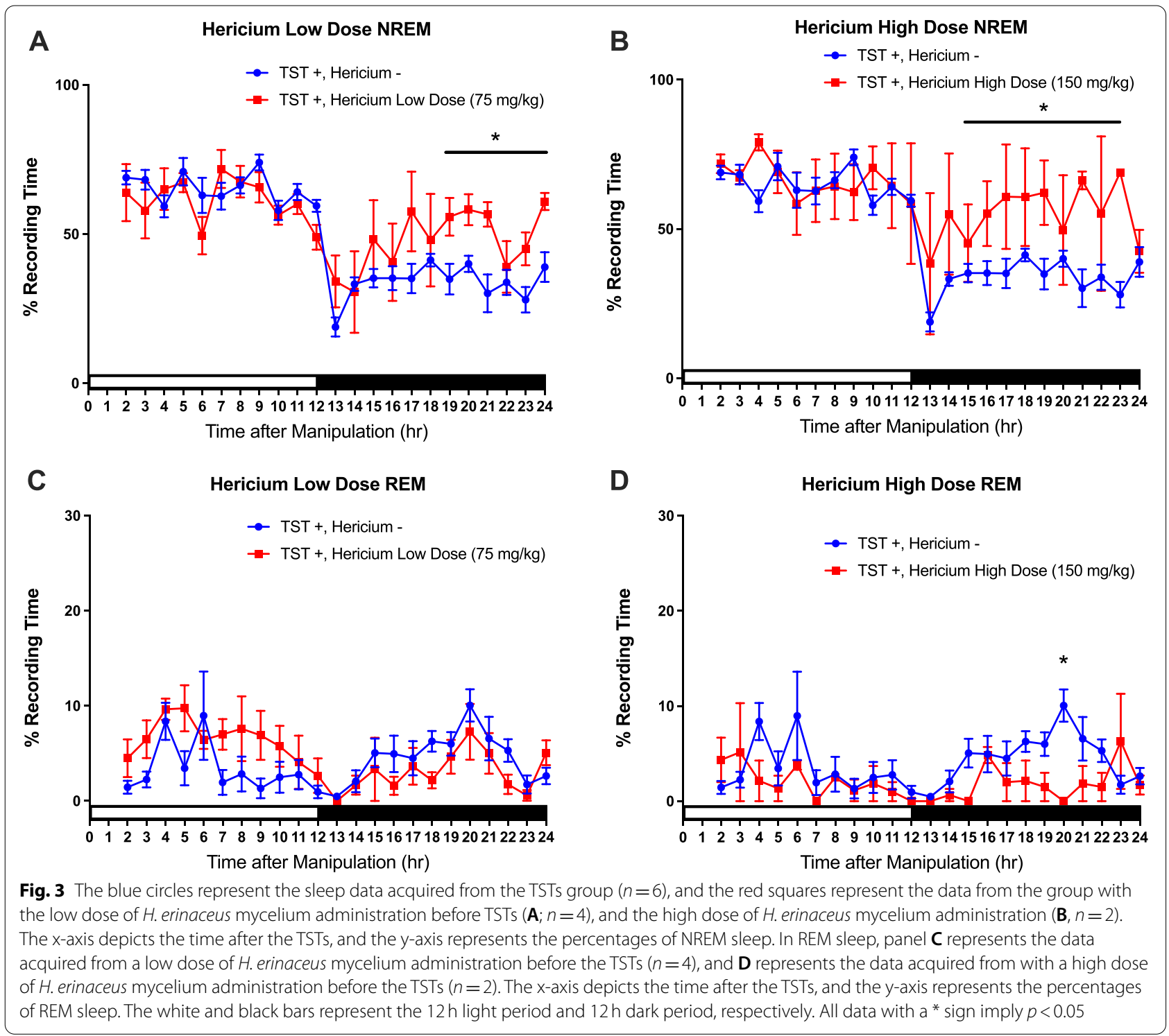

disruptions and supports healthy psychological behavior in animals.

The EPM and OF tests are comprehensive methods to analyze anxiety in mice [37]. In the previous EPM-related studies, $H$. erinaceus mycelium has shown its effectiveness in reducing anxiety [38]. However, no study has yet discussed whether $H$. erinaceus mycelium still maintains its effectiveness on the consecutive 9-day TSTs-induced anxiety and sleep disruptions. In this study, we discovered that animals with consecutive sleep disruptions and treated with TSTs spend more time in the center area and the closed arms in the EPM test. This phenomenon is reasonable because the EPM test itself stimulates the hypothalamic-pituitary-adrenal axis and sympathetic nervous system [39]. Therefore, continuous TSTs stress may cause animals' behavior to become more hyperresponsive because of the higher corticosterone levels in blood [40].

Here, we demonstrated that higher consumption of $H$. erinaceus mycelium at $150 \mathrm{mg} / \mathrm{kg}$ could significantly ameliorate anxiety levels; $75 \mathrm{mg} / \mathrm{kg}$ does not offer anxiolytic activity. To demonstrate more evidence that $H$. erinaceus mycelium can maintain its anxiolytic effect under continuous sleep disruption, the OF test is another common platform to analyze the animal's overall locomotor activity and anxiety-related behaviors [41]. Continuous TSTs do not affect the time spent in the inner area, which may be due to the habituation of 


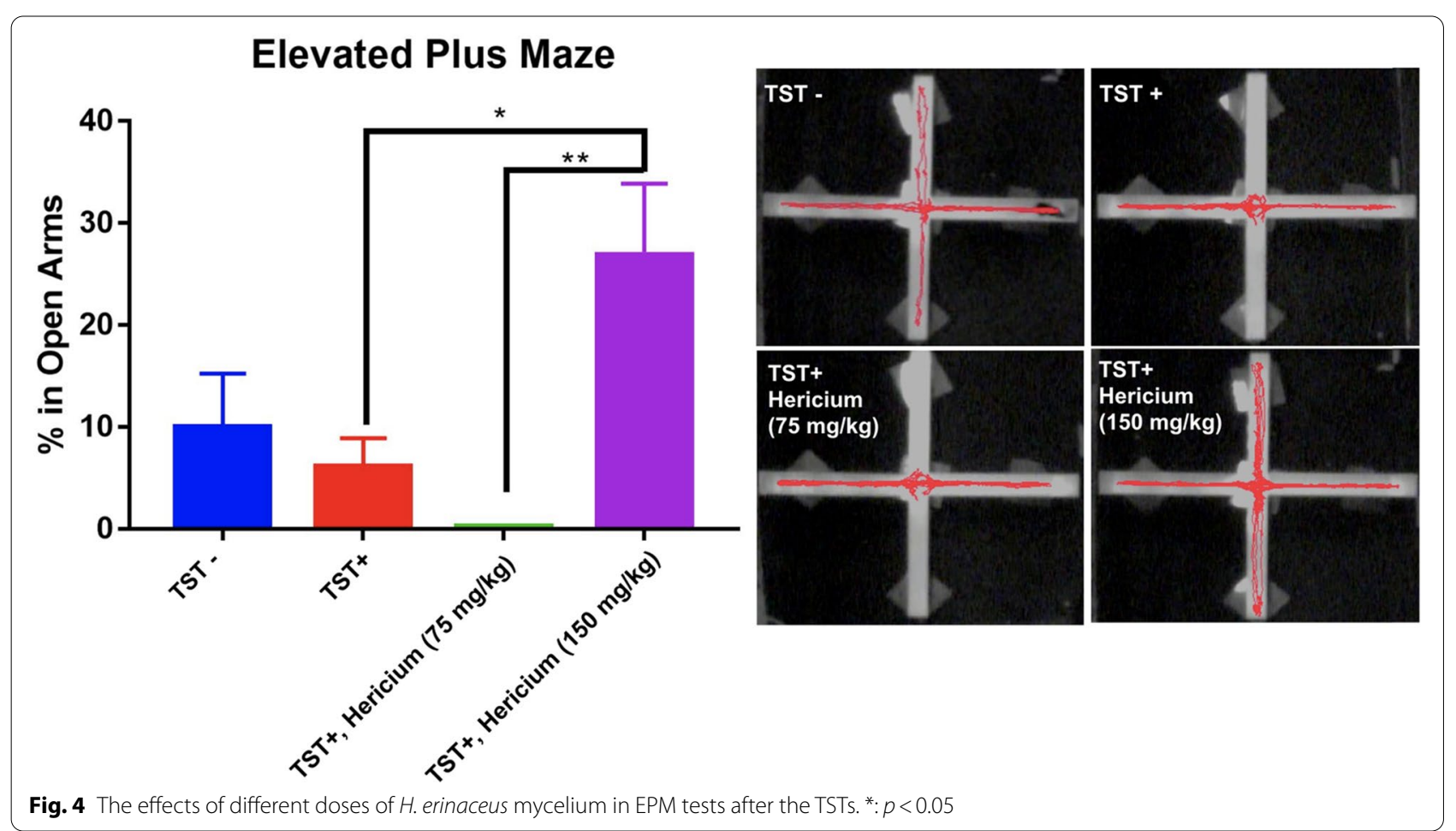

repeated handling with a subsequent decrease in heightinduced anxiety [42]. Our current study demonstrated that $150 \mathrm{mg} / \mathrm{kg} \mathrm{H}$. erinaceus mycelium can increase exploration suggesting that mice had lower anxiety than the control [43].
Dopamine is a well-known sleep-wake regulator and is closely linked with the circadian rhythm [44]. Our study found that continuous stress exposure and subsequent sleep disruptions can cause a decrease in dopamine levels. This is different from other studies

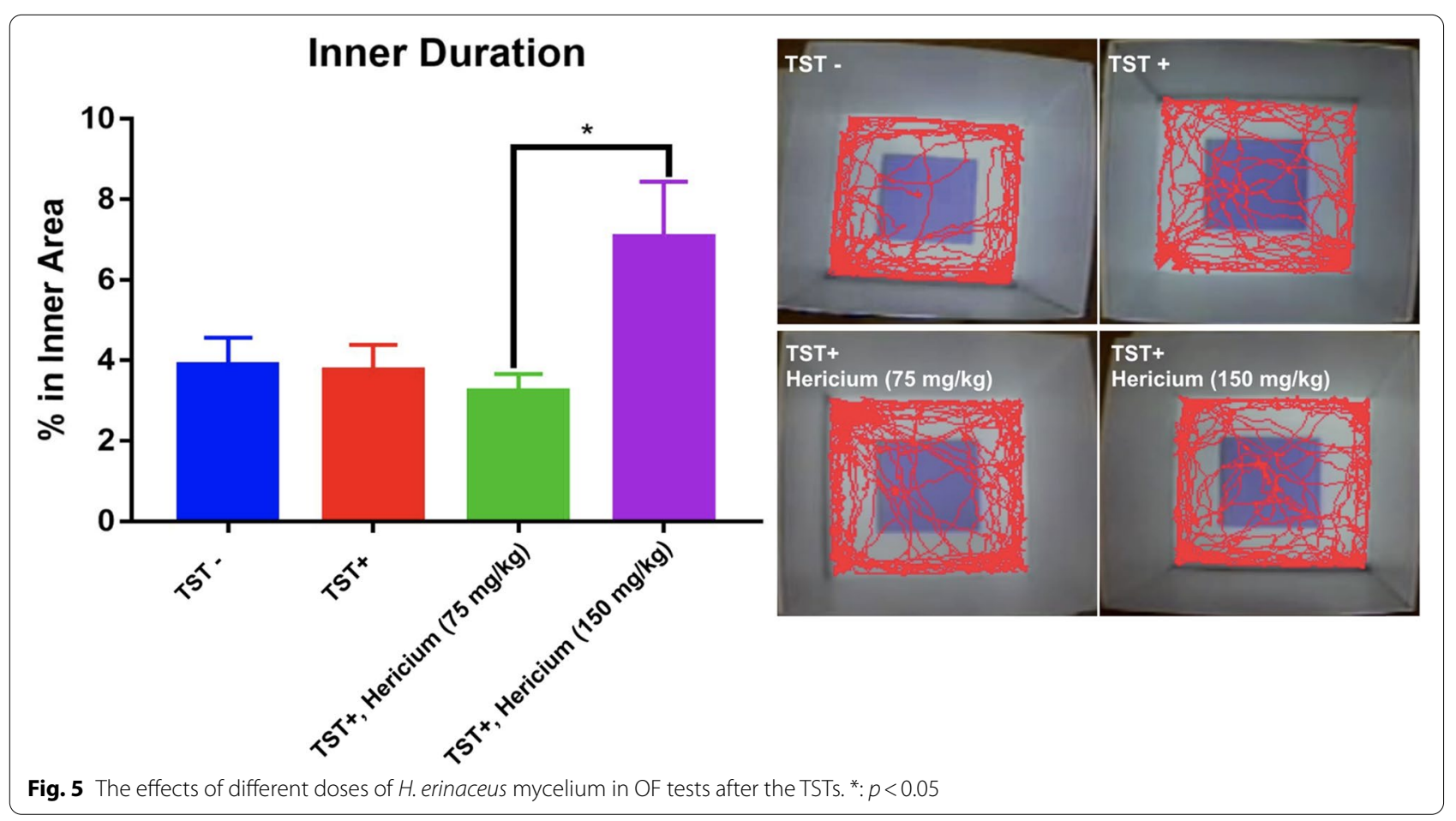




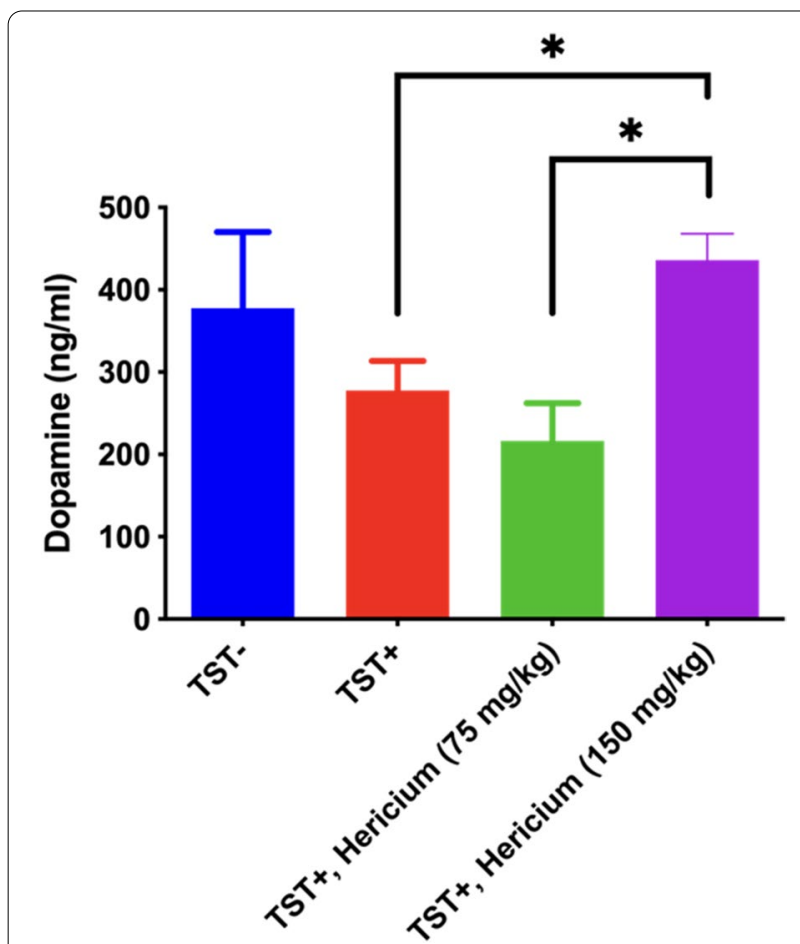

Fig. 6 The graph represents the effects of two doses of H. erinaceus mycelium in plasma dopamine concentrations. * $p<0.05$ reporting that chronic stressors cause dopaminergic blunting [45]. On the other hand, we found no difference in the levels of GABA and serotonin (data not shown). Based on these comprehensive behavioral analyses from the EPM and OFT tests, we suggest that the continuous sleep disruption induced by early anxiety from the TSTs can be ameliorated by $H$. erinaceus mycelium through the restoration of the dopamine levels. Our result also elucidated that a high dose of $H$. erinaceus mycelium involves increasing BDNF expression within the brain is an important biomarker for sleep behavioral changes. For the first time, this study also showed that erinacine $A$ and erinacine $C$ in the $H$. erinaceus mycelium can be major compounds and active ingredients. A recent clinical experiment suggested that 8 weeks of oral supplementation of $H$. erinaceus mycelium can improve mood and sleep disorders as determined by the circulating pro-BDNF and BDNF as biomarkers [46]. Future studies should address the interaction between these compounds together with BDNF neurotransmitters to better understand the dual roles of $H$. erinaceus mycelium in both sleep and anxiety. It's also important to investigate the potential factors, such as other neuroinflammatory pathway that contribute to the efficacy of $H$. erinaceus mycelium.

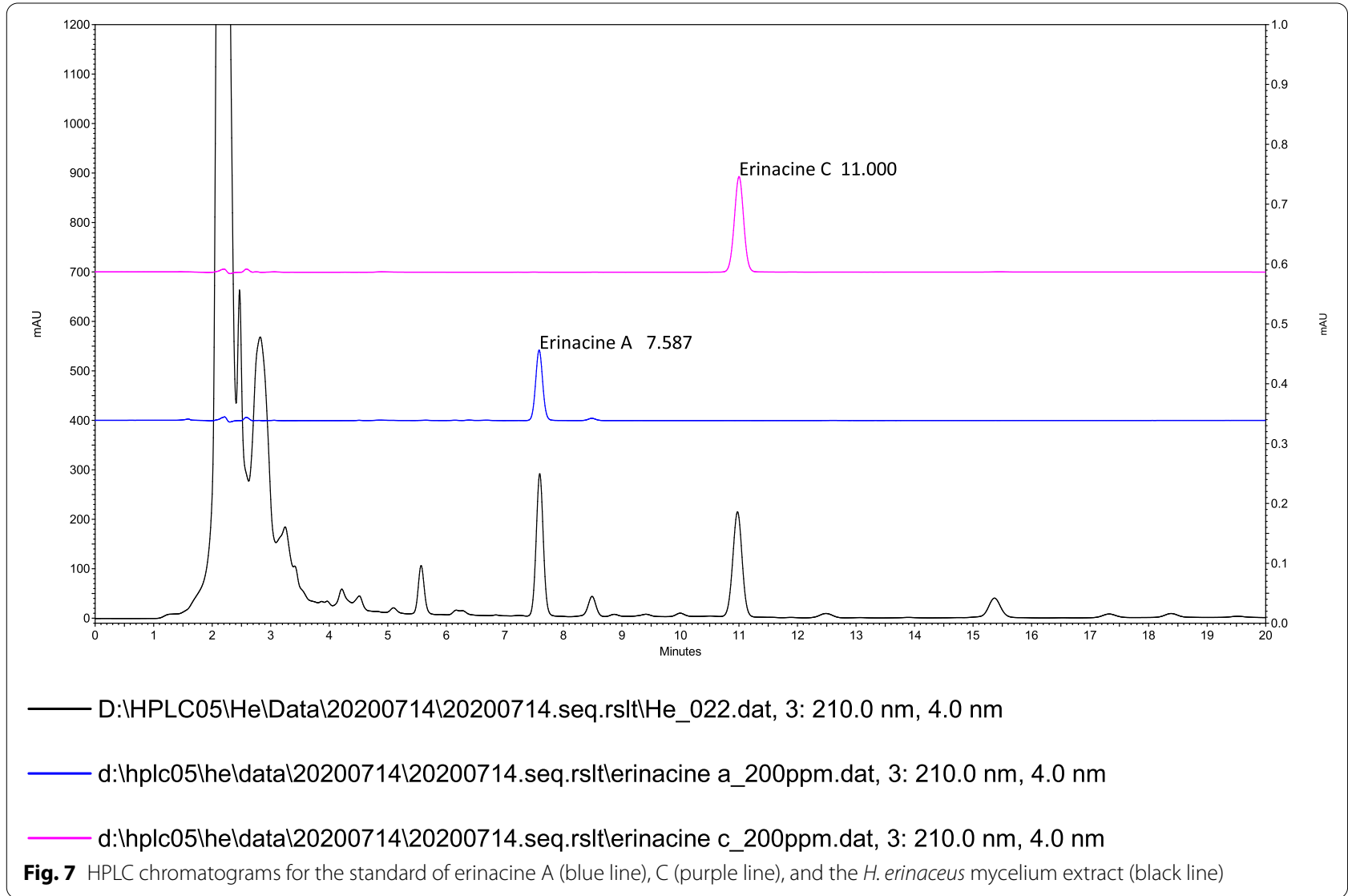




\section{Conclusion}

Our results indicated that the high dose $(150 \mathrm{mg} / \mathrm{kg})$ of $H$. erinaceus mycelium with erinacine A $7.20 \mathrm{mg} / \mathrm{g}$ and erinacine $\mathrm{C} 3.35 \mathrm{mg} / \mathrm{g}$ contents reversed the TSTinduced sleep disruptions. H. erinaceus mycelium also showed its dual potential roles in anxiety relief and sleep improvement. Future clinical trials should address these dual effects of $H$. erinaceus mycelium through a randomized placebo-controlled trial.

\begin{abstract}
Abbreviations
BDNF: Brain-derived neurotrophic factor; EEG: Electroencephalogram; EMG: Electromyogram; EPM: Elevated plus maze; H. erinaceus: Hericium erinaceus mycelium; HPLC: High-performance liquid chromatography; NGF: Nerve growth factor; OF: Open field; REM: Rapid eye movement; NREM: Non-rapid eye movement; TST: Tail suspension test.
\end{abstract}

\section{Supplementary Information}

The online version contains supplementary material available at https://doi. org/10.1186/s12906-021-03463-3.

Additional file 1: Figure S1. Unprocessed western blots images of brain tissue were provided. Each lane represents one mouse brain lysate with treatment as labeled above. The blot was cut prior to hybridization with antibodies for BDNF protein (bottom lane for mature form) and GADPH protein due to close protein band size. The blot intensities were quantified by BDNF to GAPDH expression from the same lysate lane (BDNF lane 1/ GADPH lane 1), for each treatment group total $n=3$. $: p<0.05$.

\section{Acknowledgements}

Not applicable.

\section{Authors' contributions}

Data interpretation, methodology and animal investigation was conducted by T.-Y.L., Y.L. and F.-C.C. Raw materials were provided by:T.-Y.L., L.-Y.L., I.-C.L., C.-C.C. Original draft preparation was done by T.-J.L., C.-C.C and F.-C.C. All authors have read and agreed to the published version of the manuscript.

\section{Funding}

None.

\section{Availability of data and materials}

The data and raw materials presented in this study are available from the corresponding author upon request.

\section{Declarations}

\section{Ethics approval and consent to participate}

The experimental and husbandry procedures were approved by the National Taiwan University Institutional Animal Care and Use Committee (approval number NTU-107-EL-00182) and was carried out in compliance with the ARRIVE guidelines. All methods were performed in accordance with the relevant guidelines and regulations. The experimental design was taken seriously in reducing animal numbers and suffering.

\section{Consent for publication}

Not applicable.

\section{Competing interests}

The authors have no conflicts of interest to declare.

\section{Author details}

${ }^{1}$ Biotech Research Institute, Grape King Bio, Taoyuan 32542, Taiwan. ${ }^{2}$ Department of Veterinary Medicine, National Taiwan University, Taipei, Taiwan. ${ }^{3}$ Department of Food Science, Nutrition, and Nutraceutical Biotechnology, Shih Chien University, Taipei, Taiwan. ${ }^{4}$ Institute of Food Science and Technology, National Taiwan University, Taipei, Taiwan. ${ }^{5}$ Graduate Institute of Brain and Mind Sciences, College of Medicine, National Taiwan University, Taipei, Taiwan. ${ }^{6}$ Graduate Institute of Acupuncture Science, College of Chinese Medicine, China Medical University, Taichung City, Taiwan. ${ }^{7}$ Department of Medicine, College of Medicine, China Medical University, Taichung City, Taiwan.

Received: 26 March 2021 Accepted: 15 November 2021

Published online: 05 December 2021

\section{References}

1. Consensus Conference Panel, Watson NF, Badr MS, Belenky G, Bliwise DL, Buxton OM, et al. Joint consensus statement of the American academy of sleep medicine and sleep research society on the recommended amount of sleep for a healthy adult: methodology and discussion. J Clin Sleep Med. 2015;11:931-52.

2. Xie L, Kang H, Xu Q, Chen MJ, Liao Y, Thiyagarajan M, et al. Sleep drives metabolite clearance from the adult brain. Science. 2013;342:373-7.

3. Ohayon M, Wickwire EM, Hirshkowitz M, Albert SM, Avidan A, Daly FJ, et. al. National Sleep Foundation's sleep quality recommendations: first report. Sleep Health. 2017;3:6-19.

4. Klinzing JG, Niethard N, Born J. Mechanisms of systems memory consolidation during sleep. Nat Neurosci. 2019;22:1598-610.

5. Pires GN, Bezerra AG, Tufik S, Andersen ML. Effects of acute sleep deprivation on state anxiety levels: a systematic review and meta-analysis. Sleep Med. 2016;24:109-18.

6. Sher L. COVID-19, anxiety, sleep disturbances and suicide. Sleep Med. 2020;70:124.

7. Brown RE, Basheer R, McKenna JT, Strecker RE, McCarley RW. Control of sleep and wakefulness. Physiol Rev. 2012;92:1087-187.

8. Qureshi MF, Jha SK. Short-term total sleep-deprivation impairs contextual fear memory, and contextual fear-conditioning reduces REM sleep in moderately anxious Swiss mice. Front Behav Neurosci. 2017;11:239.

9. Steiger A, Pawlowski M. Depression and sleep. Int J Mol Sci. 2019;20:607.

10. Ramos OV, Torterolo P, Lim V, Chase MH, Sampogna S, Yamuy J. The role of mesopontine NGF in sleep and wakefulness. Brain Res. 2011;1413:9-23.

11. Um MY, Yang H, Han JK, Kim JY, Kang SW, Yoon M, et al. Rice bran extract supplement improves sleep efficiency and sleep onset in adults with sleep disturbance: a randomized, double-blind, placebo-controlled, polysomnographic study. Sci Rep. 2019;9:1-8.

12. Thongbai B, Rapior S, Hyde KD, Wittstein K, Stadler M. Hericium erinaceus, an amazing medicinal mushroom. Mycol Prog. 2015;14:91.

13. Shimbo M, Kawagishi $\mathrm{H}$, Yokogoshi $\mathrm{H}$. Erinacine a increases catecholamine and nerve growth factor content in the central nervous system of rats. Nutr Res. 2005;25:617-23.

14. Rahman MA, Abdullah $N$, Aminudin N. Inhibitory effect on in vitro LDL oxidation and HMG Co-A reductase activity of the liquid-liquid partitioned fractions of Hericium erinaceus (Bull.) Persoon (lion's mane mushroom). Biomed Res Int. 2014;2014:828149.

15. Lee K-C, Kuo H-C, Shen C-H, Lu C-C, Huang W-S, Hsieh M-C, et al. A proteomics approach to identifying novel protein targets involved in erinacine A-mediated inhibition of colorectal cancer cells' aggressiveness. J Cell Mol Med. 2017;21:588-99.

16. Lee K-F, Chen J-H, Teng C-C, Shen C-H, Hsieh M-C, Lu C-C, et al. Protective effects of Hericium erinaceus mycelium and its isolated erinacine $A$ against ischemia-injury-induced neuronal cell death via the inhibition of iNOS/p38 MAPK and nitrotyrosine. Int J Mol Sci. 2014;15:15073-89.

17. Li I-C, Lee L-Y, Tzeng T-T, Chen W-P, Chen Y-P, Shiao Y-J, et al. Neurohealth properties of mycelia enriched with erinacines. Behav Neurol. 2018;2018:5802634.

18. Lee K-F, Tung S-Y, Teng C-C, Shen C-H, Hsieh MC, Huang C-Y, et al. Posttreatment with erinacine $\mathrm{a}$, a derived diterpenoid of , attenuates neurotoxicity in MPTP model of Parkinson's disease. Antioxidants (Basel). 2020;9:137. 
19. Rupcic Z, Rascher M, Kanaki S, Köster RW, Stadler M, Wittstein K. Two new cyathane diterpenoids from mycelial cultures of the medicinal mushroom hericium erinaceus and the rare species, hericium flagellum. Int J Mol Sci. 2018;19:740.

20. Chiu C-H, Chyau C-C, Chen C-C, Lee L-Y, Chen W-P, Liu J-L, et al. Erinacine A-enriched hericium erinaceus mycelium produces antidepressant-like effects through modulating BDNF/PI3K/Akt/GSK-3 $\beta$ signaling in mice. Int J Mol Sci. 2018;19:341.

21. Hu J-H, Li I-C, Lin T-W, Chen W-P, Lee L-Y, Chen C-C, et al. Absolute bioavailability, tissue distribution, and excretion of erinacine $S$ in hericium erinaceus mycelia. Molecules. 1624;2019:24.

22. Li I-C, Chang H-H, Lin C-H, Chen W-P, Lu T-H, Lee L-Y, et al. Prevention of early Alzheimer's disease by erinacine A-enriched mycelia pilot doubleblind placebo-controlled study. Front Aging Neurosci. 2020;12:155.

23. Ratto D, Corana F, Mannucci B, Priori EC, Cobelli F, Roda E, et al. Improves recognition memory and induces hippocampal and cerebellar neurogenesis in frail mice during aging. Nutrients. 2019;11:715.

24. Li I-C, Chen Y-L, Chen W-P, Lee L-Y, Tsai Y-T, Chen C-C, et al. Genotoxicity profile of erinacine A-enriched mycelium. Toxicol Rep. 2014;1:1195-201.

25. Wang L-Y, Huang C-S, Chen Y-H, Chen C-C, Chen C-C, Chuang C-H. Anti-inflammatory effect of erinacine $\mathrm{C}$ on $\mathrm{NO}$ production through down-regulation of NF-KB and activation of Nrf2-mediated HO-1 in BV2 microglial cells treated with LPS. Molecules. 2019;24:3317.

26. El Yacoubi M, Bouali S, Popa D, Naudon L, Leroux-Nicollet I, Hamon M, et al. Behavioral, neurochemical, and electrophysiological characterization of a genetic mouse model of depression. Proc Natl Acad Sci U S A. 2003;100:6227-32.

27. Chang FC, Opp MR. Blockade of corticotropin-releasing hormone receptors reduces spontaneous waking in the rat. Am J Phys. 1998;275:R793-802.

28. Hafner M, Stepanek M, Taylor J, Troxel W, Stolk C. Why sleep matters: the economic costs of insufficient sleep. Rand Health Q. 2017;6:11.

29. Chattu VK, Manzar MD, Kumary S, Burman D, Spence DW, Pandi-Perumal SR. The global problem of insufficient sleep and its serious public health implications. Healthcare (Basel). 2018;7:1.

30. Qiu J, Shen B, Zhao M, Wang Z, Xie B, Xu Y. A nationwide survey of psychological distress among Chinese people in the COVID-19 epidemic: implications and policy recommendations. Gen Psychiatr. 2020;33:e100213.

31. Lai J, Ma S, Wang Y, Cai Z, Hu J, Wei N, et al. Factors associated with mental health outcomes among health care workers exposed to coronavirus disease 2019. JAMA Netw Open. 2020;3:e203976.

32. Amara I, Scuto M, Zappalà A, Ontario ML, Petralia A, Abid-Essefi S, et al. Hericium erinaceus prevents DEHP-induced mitochondrial dysfunction and apoptosis in PC12 cells. Int J Mol Sci. 2020;21:2138.

33. Chong PS, Fung M-L, Wong KH, Lim LW. Therapeutic potential of hericium erinaceus for depressive disorder. Int J Mol Sci. 2019;21:163.

34. Hines DJ, Schmitt LI, Hines RM, Moss SJ, Haydon PG. Antidepressant effects of sleep deprivation require astrocyte-dependent adenosine mediated signaling. Transl Psychiatry. 2013;3:e212.

35. Fultz NE, Bonmassar G, Setsompop K, Stickgold RA, Rosen BR, Polimeni $J R$, et al. Coupled electrophysiological, hemodynamic, and cerebrospinal fluid oscillations in human sleep. Science. 2019;366:628-31.

36. Machado RB, Rocha MR, Suchecki D. Brain prolactin is involved in stressinduced REM sleep rebound. Horm Behav. 2017:89:38-47.

37. Carola V, D'Olimpio F, Brunamonti E, Mangia F, Renzi P. Evaluation of the elevated plus-maze and open-field tests for the assessment of anxietyrelated behaviour in inbred mice. Behav Brain Res. 2002;134:49-57.

38. Limanaqi F, Biagioni F, Busceti CL, Polzella M, Fabrizi C, Fornai F. Potential antidepressant effects of scutellaria baicalensis, hericium erinaceus and rhodiola rosea. Antioxidants. 2020;9:234.

39. Biedermann SV, Biedermann DG, Wenzlaff F, Kurjak T, Nouri S, Auer MK, et al. An elevated plus-maze in mixed reality for studying human anxietyrelated behavior. BMC Biol. 2017;15:125.

40. Reis-Silva TM, Sandini TM, Calefi AS, Orlando BCG, Moreira N, Lima APN, et al. Stress resilience evidenced by grooming behaviour and dopamine levels in male mice selected for high and low immobility using the tail suspension test. Eur J Neurosci. 2019;50:2942-54.

41. Shoji H, Takao K, Hattori S, Miyakawa T. Age-related changes in behavior in C57BL/6J mice from young adulthood to middle age. Mol Brain. 2016:9:1-18.
42. Ueno H, Takahashi Y, Suemitsu S, Murakami S, Kitamura N, Wani K, et al. Effects of repetitive gentle handling of male $\mathrm{C} 57 \mathrm{BL} / 6 \mathrm{NCrl}$ mice on comparative behavioural test results. Sci Rep. 2020;10:1-13.

43. Wozniak DF, Valnegri P, Dearborn JT, Fowler SC, Bonni A. Conditional knockout of UBC 13 produces disturbances in gait and spontaneous locomotion and exploration in mice. Sci Rep. 2019;9:1-14.

44. Eban-Rothschild A, Appelbaum L, de Lecea L. Neuronal mechanisms for sleep/wake regulation and modulatory drive. Neuropsychopharmacology. 2018;43:937-52.

45. Holly EN, Miczek KA. Ventral tegmental area dopamine revisited: effects of acute and repeated stress. Psychopharmacology. 2016;233:163-86.

46. Vigna L, Morelli F, Agnelli GM, Napolitano F, Ratto D, Occhinegro A, et al. Hericium erinaceus improves mood and sleep disorders in patients affected by overweight or obesity: could circulating pro-BDNF and BDNF be potential biomarkers? Evid Based Complement Alternat Med. 2019;2019:1-12.

\section{Publisher's Note}

Springer Nature remains neutral with regard to jurisdictional claims in published maps and institutional affiliations.

Ready to submit your research? Choose BMC and benefit from:

- fast, convenient online submission

- thorough peer review by experienced researchers in your field

- rapid publication on acceptance

- support for research data, including large and complex data types

- gold Open Access which fosters wider collaboration and increased citations

- maximum visibility for your research: over $100 \mathrm{M}$ website views per year

At BMC, research is always in progress.

Learn more biomedcentral.com/submissions 